\title{
Disfunción de articulación sacroiliaca
}

\section{Sacroiliac joint dysfunction}

\author{
Javier Camacho-Galindo, ${ }^{*}$ Alejandro Guillermo Gallegos Tejeda ${ }^{\ddagger}$
}

Citar como: Camacho-Galindo J, Gallegos TAG. Disfunción de articulación sacroiliaca. An Med ABC. 2021; 66 (2): 120-127. https:// dx.doi.org/10.35366/100481

\section{RESUMEN}

La disfunción de la articulación sacroiliaca es uno de los múltiples diagnósticos diferenciales de lumbalgia, representando el $15-30 \%$ de la misma, y que trae como resultado afección del estilo y calidad de vida por dolor e incapacidad. Se realiza esta revisión para dar una descripción de la anatomía, presentación clínica, exploración física para orientación al diagnóstico, pruebas diagnósticas utilizadas o con posibilidad de utilizarse, y tratamiento específico y adecuado a esta entidad, que ya es subdiagnosticada. Todo con el fin de dar un instrumento más a aquellos médicos interesados para el bien de los pacientes.

Palabras clave: Articulación sacroiliaca, inyecciones diagnósticas, pelvis.

Nivel de evidencia: III

\section{INTRODUCCIÓN}

La disfunción de articulaciones sacroiliacas de origen no inflamatorio es uno de los múltiples diagnósticos diferenciales de lumbalgia, y trae como resultado afección del estilo y calidad de vida de los pacientes de la

\footnotetext{
* Ortopedia y Traumatología, Cirugía Articular y de Cadera.

‡ Residente de tercer año en Ortopedia y Traumatología.
}

\section{Centro Médico ABC. Ciudad de México.}

\section{Correspondencia:}

Dr. Javier Camacho Galindo

E-mail: jvrcamacho@hotmail.com

Recibido: 02/04/2021. Aceptado: 08/06/2021.

\begin{abstract}
The sacroiliac joint dysfunction is one of the multiple etiologies of lower back pain, being the 15-30\% of back pain, which brings changes in the quality and style of living duet to pain and disability. This revision gives us a description of the anatomy involved, clinical presentation, physical examination with orientation to the diagnosis, diagnostic maneuvers used or with the possibility of using, and specific and adequate treatment of this entity, which it is already underdiagnosed. All done with the end for giving an instrument to those physicians interested in well-being of the patients.
\end{abstract}

Keywords: Sacroiliac joint, diagnostic injections, pelvis.

Level of evidence: III

misma intensidad que la osteoartrosis de cadera, rodilla, espondilolistesis degenerativa y estenosis espinal. ${ }^{1}$ La dificultad para el diagnóstico radica en la sobreposición de signos y síntomas que orientan a múltiples etiologías. ${ }^{2}$ En esto radica la importancia de entender esta entidad, para su diagnóstico y tratamiento adecuado y orientado al origen. ${ }^{1}$ Además, en cualquier lumbalgia debe considerarse que la articulación sacroiliaca puede o no estar involucrada en el cuadro clínico. ${ }^{3}$

\section{EPIDEMIOLOGÍA Y ETIOLOGÍA}

La lumbalgia de origen sacroiliaco se ha reportado en un $15-30 \%$. El origen puede ser degenerativo, inflamatorio, artrosis, lesión traumática o embarazo. Se ha descrito en estudios un aumento del porcentaje en pacientes sometidos a fusión lumbar. ${ }^{4}$ 
Palsson refiere el dolor de articulaciones sacroiliacas y las engloba en tres etiologías principales: del embarazo, de enfermedad específica y dolor lumbar sacroiliaco no específico. ${ }^{3}$

\section{Anatomía y biomecánica}

La articulación sacroiliaca es una articulación diartrodial irregular. Por ello, está cubierta de cartílago hialino en ambas superficies contenida en una cápsula articular. En la porción posterior está limitada principalmente por un ligamento complejo y grueso, logrando limitar el movimiento. ${ }^{4}$

La articulación se encuentra dentro de los segmentos sacros S1-S3 de forma cóncava, mientras que la del iliaco es predominantemente de forma convexa en su superficie articular.

La superficie articular se divide en tres porciones, correspondientes a cada vértebra sacra: craneal, media y caudal, siendo la cefálica la más grande y la caudal la más chica. Sin embargo, cuando el paciente está en bipedestación, la porción craneal está más ventral y la caudal está dorsal, por lo que sería más correcto ser identificadas de esta forma. ${ }^{5}$

Thawrani y colaradores reportan que la cápsula posterior es rudimentaria y prácticamente es únicamente estructuras ligamentarias. Esto explicaría que, al no estar rodeada por completo de cápsula articular, los marcadores inflamatorios que inician en la articulación se extravasan hacia los ramos dorsales y provocan dolor que puede mimetizar o venir acompañado de dolor radicular. ${ }^{2}$

La cápsula posterior está inervada por las ramas laterales de las raíces posteriores de L5-S4 con fibras sensitivas y nociceptivas, por lo que pueden causar dolor. ${ }^{4}$

La inervación anterior suele ser más ambigua, con reporte de que es dada por L5-S2, aunque antes se creía que era por el nervio glúteo superior y obturador. ${ }^{6}$

Es una articulación que transmite la carga del esqueleto axial al apendicular, a ambas extremidades pélvicas. ${ }^{4}$

Por lo mismo, la articulación debe ser suficientemente fuerte y estable para poder realizar una adecuada transmisión de cargas. ${ }^{4}$ Así como disipación de cargas del tronco hacia las extremidades inferiores. ${ }^{2}$

El movimiento que realiza la articulación sacroiliaca se describe como nutación y contranutación, ${ }^{4}$ que se refieren a la combinación de rotación y traslación de la articulación en conjunto. ${ }^{5}$ La nutación implica inclinación del sacro respecto al iliaco al nivel de S2 como eje transverso, con acentuación de la lordosis lumbar.
Se debe tener en cuenta que esta articulación se mueve en los tres ejes. En el eje sagital el movimiento varía de $1-4^{\circ}$. Se incluye una traslación de aproximadamente 0.5-2 $\mathrm{mm}$.

Existen múltiples variantes anatómicas entre sexo masculino y femenino en pelvis y en la articulación sacroiliaca, incluyendo las ligamentarias. En las mujeres, los ligamentos son más laxos para permitir el parto. ${ }^{2}$ Los ligamentos involucrados en la estabilidad de la articulación son: el sacroiliaco anterior, el sacroiliaco posterior, el sacroespinoso, el sacrotuberoso y los interóseos.

Los ligamentos a su vez actúan de forma conjunta para la movilidad y estabilidad de esta articulación con los músculos gluteus maximus, gluteus medius, iliopsoas, piriformis, bíceps femoris, oblicuos abdominales, transverso del abdomen y la fascia toracolumbar. ${ }^{1,6}$

La porción ventral superior está cubierta por el ligamento iliolumbar, por la banda lumbosacra del ligamento. El resto de la porción anterior es el ligamento sacroiliaco anterior. El ligamento sacroespinoso, en su porción cefálica, se fusiona con la cápsula sacroiliaca en su región caudal. El ligamento interóseo sacroiliaco es el más fuerte de los ligamentos homónimos; sin embargo, el ligamento sacroiliaco posterior es el ligamento más relacionado con la movilidad de la articulación. El eje de rotación de la articulación está al nivel de los ligamentos sacroiliacos interóseos. ${ }^{5}$

El complejo ligamentario posterior es más complejo que el ventral. Se divide en capa superficial y profundo. El ligamento sacroiliaco dorsal se origina de la cresta sacra y se inserta en la espina iliaca posterosuperior.

El ligamento iliolumbar o lumbosacro va de las apófisis transversas de L4 y L5 y se dirige hacia la cresta iliaca y la cápsula articular de la sacroiliaca ipsilateral. La principal función de este ligamento es limitar el movimiento de la unión lumbosacra. ${ }^{5}$

\section{Presentación clínica}

El dolor lumbar es parte de la presentación clínica, la causa es compleja de aislar e identificar. ${ }^{1} \mathrm{El}$ paciente suele referir dolor que varía con la posición o al hacer cambio de la posición. Suele haber mayor molestia al estar parado o sentado y que remite parcial o de forma completa al recostarse o al caminar. Es frecuente que el dolor sea referido hacia región inguinal o cefálica a L5, rara vez será por alteración en articulaciones sacroiliacas de forma aislada. ${ }^{1}$ 
Aunque hay otros estudios donde el dolor inguinal sí debe ser considerado como posible origen sacroiliaco. ${ }^{4}$

Como menciona Schneider y colaboradores, no hay patrones de dolor referido o datos claves en la historia del paciente que orienten directa y únicamente a afección de articulación sacroiliaca. ${ }^{7}$

Sin embargo, esto se debe a la alta variabilidad de presentación de lesión de articulación sacroiliaca; el patrón más común que nos puede orientar a ello, sin ser patognomónico, es el dolor en nalga ipsilateral hasta el muslo posterolateral. También se ha visto correlación entre el dolor a $10 \mathrm{~cm}$ caudales y $3 \mathrm{~cm}$ laterales a la espina iliaca posterosuperior. ${ }^{2,6}$

El origen del dolor tiene que tomar en cuenta la posibilidad de que se trate de un dolor de origen facetario lumbar, de piriformis, de la fascia y musculatura dorso lumbar y dolor discogénico, entre otros. ${ }^{4}$

La patología de cadera, alteraciones gastrointestinales, genitourinarias o hasta ginecológicas se encuentran dentro del espectro de posibilidades, que puedan mimetizar la misma historia clínica de afección de sacroiliacas. ${ }^{2}$

En un estudio de revisión por Cohen, la presentación clínica de disfunción de articulación sacroiliaca se puede presentar de forma múltiple. Las posibilidades son dolor en región glútea, se presenta en $94 \%$; $72 \%$ como lumbalgia, con extensión hacia el muslo en un 50\%; el dolor debajo de la rodilla se presenta en $28 \%$ de los pacientes y sólo un $14 \%$ con dolor inguinal; otro $14 \%$ presenta el dolor referido hasta el pie. De igual forma, concluye que los patrones son múltiples y no siguen un patrón único o específico. ${ }^{2,6,8}$

Adormecimiento y/o sensación de clic son también posibles patrones de dolor de origen sacroiliaco. Refieren que el dolor rara vez es cefálico a la línea del cinturón. ${ }^{9}$

\section{Exploración física}

Al no tener una presentación clínica completamente dirigida a la disfunción de articulación sacroiliaca, obtener un diagnóstico certero se dificulta. El diagnóstico principalmente se logra tras infiltración de la articulación y observar mejoría de la sintomatología. Sin embargo, no todos los médicos están entrenados para realizarlo, por lo que la exploración física siempre es requerida para orientar al médico a un diagnóstico certero.

En múltiples estudios, la exploración física se resume a 5-6 pruebas físicas especiales. La fortaleza de realizarlas radica en aumentar la sensibilidad, especificidad $\mathrm{y}$ valores predictivos al tener varias pruebas positivas.
Nejati realizó un estudio para determinar la sensibilidad, especificidad y valores predictivos de pruebas subjetivas y de movilidad para disfunción de articulación sacroiliaca. ${ }^{10}$

\section{Pruebas de movimiento}

1. Prueba de Gillet: el examinador se encuentra detrás del paciente y coloca un pulgar en la espina iliaca posterosuperior del lado que se busca examinar y el otro pulgar en el sacro. Al paciente se le solicita que flexione y lleve la rodilla ipsilateral de la espina iliaca que se palpa al tórax. Se realiza de forma bilateral. La prueba se considera positiva si el pulgar en la espina iliaca se mantiene en la misma altura o se eleva. Es negativa cuando el pulgar baja. ${ }^{10}$

2. Prueba de flexión anterior: con ambos pulgares en cada espina iliaca posterosuperior, se solicita al paciente que flexione las caderas hasta el máximo del movimiento. Una prueba positiva se refiere cuando hay movimiento hacia cefálico de algún pulgar, siendo significativo de hipomovilidad de la articulación ipsilateral. ${ }^{10}$

\section{Pruebas de provocación}

1. Prueba de Patrick: el paciente deberá estar en decúbito supino. El examinador debe colocar la cadera en flexión, abducción y rotación externa, con la rodilla flexionada a $90^{\circ}$ y con el maléolo lateral sobre la rodilla contralateral. Se debe realizar presión hacia la mesa de la rodilla flexionada y de la cresta iliaca anterosuperior contralateral. Se considera positiva si hay dolor en región de articulación sacroiliaca. Dolor en región inguinal indica más una patología de la cadera. ${ }^{10}$

2. Prueba de cizallamiento posterior: con paciente en decúbito supino, el examinador flexiona la cadera y rodilla a $90^{\circ}$. Posteriormente realiza carga axial sobre el eje femoral. Si presenta dolor en articulación sacroiliaca ipsilateral, se considera positiva. ${ }^{10}$

3. Prueba de Gaenslen: el paciente se encuentra en decúbito supino con una pierna colgando del borde de la mesa y la otra con flexión máxima de cadera y rodilla hacia el tórax. El examinador realiza presión contra la rodilla en flexión y la rodilla en la mesa. Se considera positivo si hay dolor en la pierna que cuelga. ${ }^{10}$

4. Prueba de Yeoman: se le solicita al paciente colocarse en decúbito prono. El examinador flexiona la rodilla a $90^{\circ}$ con una mano. Con el codo libre 
del examinador, se estabiliza la pelvis. Se realiza extensión de la cadera con la mano en la rodilla. Si hay dolor, se considera una prueba positiva. ${ }^{10}$

5. Prueba de compresión: con el paciente en decúbito lateral, se realiza compresión de la cresta iliaca y espina iliaca anterosuperior hacia la mesa. Si hay dolor, se considera prueba positiva. ${ }^{10}$

6. Prueba de distracción: se solicita al paciente colocarse en decúbito supino. Se realiza compresión al mismo tiempo de espinas iliacas anterosuperiores. El dolor determina la positividad de la prueba. ${ }^{10}$

7. Prueba de Fortin: se le solicita al paciente que determine el sitio de dolor. Es positivo cuando se encuentra el epicentro del dolor en los primeros $2 \mathrm{~cm}$ posteromediales a la espina iliaca posterosuperior. $^{2}$

En el estudio realizado por Nejati y su equipo, las pruebas con una sensibilidad de $100 \%$ fueron el test de Gillet y el test de flexión anterior de cadera, ambas pruebas de movilidad. En cuanto a pruebas de provocación de dolor de articulación sacroiliaca, la prueba de cizallamiento posterior fue la más sensible con $74.4 \%$, también referida como la más sensible por Schneider. ${ }^{7}$

La prueba más específica fue el test de Patrick con $66.7 \%$. Las pruebas de movimiento obtuvieron $0 \%$ de especificidad. El resto de las pruebas de provocación analizadas y reportadas no superaban el $50 \%$ de especificidad. De igual forma, la prueba de Patrick fue la que obtuvo un valor predictivo positivo de $90.3 \%$ siendo la más alta en comparación con otras pruebas. ${ }^{10}$

Se ha concluido en múltiples estudios que para una adecuada sensibilidad, especificidad y valores predictivos, la disfunción de la articulación sacroiliaca se debe sospechar cuando hay tres o más pruebas positivas. De esta forma se logra una sensibilidad y especificidad mayores. ${ }^{1,2,6,9-12}$

Laslett refiere que tres o más pruebas provocativas o tener dos pruebas positivas de cuatro, son predictores positivos para un bloqueo intraarticular. ${ }^{11}$

\section{Estudios de imagen}

Debemos recordar que la etiología de la alteración de articulación sacroiliaca es múltiple. La radiografía y la resonancia magnética son estudios que ayudan al diagnóstico de la sacroileítis de origen inflamatorio, infeccioso, fracturas, neoplasias, etcétera. Sin embargo, en la disfunción de la articulación sacroiliaca, rara vez se obtiene información adecuada en los estudios de gabinete, ${ }^{1,2}$ que ayudan a identificar esclerosis, erosiones o anquilosis de la articulación. ${ }^{12,13}$

La tomografía computarizada (TC) es de igual forma un estudio con baja sensibilidad para el diagnóstico de disfunción de articulación sacroiliaca. ${ }^{6}$

Algunas infecciones, osteítis condensans ilii, espondilitis anquilosante, gota, entre otras patologías se benefician más con radiografías, $\mathrm{TC}$ y resonancia magnética que la disfunción de la articulación sacroiliaca. ${ }^{12}$

Dismorfismos congénitos o fracturas son entidades que deben descartarse al tomar estudios de gabinete, pero no dan el diagnóstico definitivo. ${ }^{4}$

\section{Inyecciones intraarticulares diagnósticas}

Al no tener una presentación clínica y examen físico directos y claros para determinar el diagnóstico de disfunción de articulación sacroiliaca, las inyecciones intraarticulares son el método más efectivo del que se tiene recurso, sin ser considerado el estándar de oro. ${ }^{1,9}$ Además de funcionar como prueba diagnóstica, también tiene su función terapéutica. ${ }^{2}$

Una cantidad considerable de estudios acuerdan que, si el dolor es controlado más del $75 \%$, es de origen sacroiliaco. ${ }^{1,2} \mathrm{Si}$ el control del dolor es del 50 $74 \%$, el origen es multifactorial. Y si es menor a $50 \%$, el origen del dolor no es sacroiliaco. ${ }^{13}$

Las inyecciones deben realizarse bajo control fluoroscópico, como es referido por Schneider. ${ }^{14}$ Aunque se ha descrito por Foley la posibilidad de realizarlas bajo control en tomografía, resonancia magnética o ultrasonido. Ellos mismos mencionan que si no hay control de imagen sólo el $22 \%$ será intraarticular. ${ }^{2,9}$

Peebles y colaboradores reportan una precisión variable cuando el control es realizado con ultrasonido de 40-90\%. Para el control fluoroscópico, da una precisión de 97-98\%. Para el control con tomografía computarizada da una precisión de $100 \%$. Por lo que concluye que cualquier modalidad de las últimas dos es una buena opción para una adecuada inyección intraarticular. ${ }^{12}$

Nejati y su equipo, entre otros, sugieren realizar la inyección intraarticular con abordaje de doble bloqueo. A pesar de que esté descrito de bloqueo único como una posibilidad, el doble bloqueo disminuye la prevalencia de disfunción de articulación sacroiliaca. ${ }^{10}$

Así se ha visto disminución de la incidencia de falsos positivos cuando se realiza un doble bloqueo, comparado con el bloqueo único. ${ }^{7}$ 
Por el contrario, Simopoulos y su grupo realizan una revisión donde identifican una baja prevalencia con bloqueo único. Sobre todo cuando el dolor remite aproximadamente en un $90 \%$. Sin embargo, hay estudios citados y analizados en esta revisión con prevalencia de $30 \%$ cuando el punto de corte disminuye a $75 \%$. De la misma forma, el estudio concluye que las inyecciones como un método diagnóstico tienen un nivel de evidencia II-III. ${ }^{15}$

Las inyecciones intraarticulares pueden ser de lidocaína, bupivacaína, triamcinolona o betametasona como lo refiere $\mathrm{Ou}$-Yang y colaboradores en su artículo. ${ }^{1}$

Los pacientes a los que de forma clínica se les diagnostica disfunción de la articulación sacroiliaca con tres pruebas físicas o más positivas son los que tendrán un resultado exitoso diagnóstico y terapéutico. El valor predictivo positivo de estos casos es reportado en $77 \%$ y un valor predictivo negativo en $87 \% .^{1}$

De igual forma, una infiltración con mucho volumen puede causar falsos positivos para disfunción de articulación sacroiliaca debido al flujo de los medicamentos hacia estructuras posteriores, que como recordaremos en el repaso anatómico, la cápsula posterior es principalmente reemplazada por ligamentos y no hay una adecuada contención posterior. ${ }^{2}$

$\mathrm{Al}$ realizar la inyección intraarticular, se debe tomar un control con contraste para poder determinar que se encuentra realmente intraarticular y evitar infiltración intravascular. Después con corroboración fluoroscópica se inyecta la combinación de analgésico y de corticoesteroide. ${ }^{9}$

Los medicamentos que se pueden infiltrar son 0.5-1\% de lidocaína, $0.25 \%$ de levobupivacaína, $10 \mathrm{mg}$ de dexametasona, $40 \mathrm{mg}$ de triamcinolona o $40 \mathrm{mg}$ de metilprednisolona. ${ }^{12}$ Tanto Foley como Thawrani refieren el límite de infiltración en 2-2.5 mL. ${ }^{2,9}$

La técnica para el control fluoroscópico, como es referido en el artículo de Soto Quijano, es la técnica de Hendrix. El paciente se encuentra en decúbito prono y se toma control fluoroscópico de la pelvis con inclinación caudocraneal. Se ajusta lateral o medialmente $5^{\circ}$ hasta obtener una sobreposición del espacio articular inferior anterior y posterior. En la parte inferior de la articulación, se introduce $3 \mathrm{~cm}$ la aguja de $22 \mathrm{~g}$. Se debe corroborar con una proyección lateral un adecuado trayecto y después se continúa introduciendo la aguja con proyecciones anteroposteriores hasta encontrarse intraarticular. Se introduce material de contraste en el que debe verse que se recorre hacia la parte superior de la articulación, cuando se está verdaderamente intraarticular. La importancia de esta migración radica en que a las dos semanas hay una reducción del dolor mayor. ${ }^{13}$
Aunque hay nuevas técnicas para acceso intraarticular de la articulación sacroiliaca, como el referido por Taheri y su grupo, ellos mismos concluyen que no hay diferencia con otras técnicas de infiltración, siempre y cuando el patrón de distribución del contraste fuera adecuado. De igual forma, no hay diferencia en lado afectado y género del paciente. ${ }^{16}$

\section{Tratamiento}

El tratamiento conservador es el primer escalón, que consiste en la realización de terapia física con enfoque al fortalecimiento de tronco. Dar la fortaleza y empezar protocolos de flexibilidad busca corregir el desbalance mal adaptativo y biomecánico que los pacientes puedan tener. La terapia física deberá ser personalizada. ${ }^{6}$

Dentro del tratamiento conservador, también se encuentra el uso de cinturones, manipulación manual y las inyecciones periódicas. ${ }^{17}$

Además de lo ya mencionado, en la terapia física deberá haber reeducación y entrenamiento biomecánico y de la postura del paciente. El fortalecimiento del paciente estará enfocado a los músculos isquiotibiales, gluteus maximus, gluteus medius, piriformis, erector spinae, latissimus dorsi e iliacos. ${ }^{18}$

Dentro de los medicamentos para controlar el dolor se encuentran los antiinflamatorios no esteroideos (AINE), analgésicos no opiáceos, opioides y antidepresivos entre otros. El etanercept es un medicamento utilizado cuando la alteración sacroiliaca es de origen inflamatorio reumático y no está dentro del cuadro de medicamentos de la disfunción de articulación sacroiliaca. ${ }^{18}$

Se realizó un estudio comparativo entre la terapia de manipulación y los ejercicios de estabilización por Kamali. A pesar de la alta cantidad de abandono del tratamiento, no hubo diferencia significativa entre las terapias realizadas. ${ }^{19}$ Como terapia adyuvante, Peebles menciona la posibilidad de la acupuntura y yoga como opciones.

De igual forma refiere que si la lesión es aguda, los primeros tres días se manejen con hielo, antinflamatorios y reposo relativo. Una vez controlado el dolor, se debe iniciar el manejo del desbalance muscular, ya que un desequilibrio entre músculos antagonistas, pueden mantener una inclinación pélvica inadecuada. Además puede ser causa de un acortamiento funcional de la extremidad si la afección es unilateral. ${ }^{12}$

En los atletas suele haber contracturas de los aductores de la cadera, obturator internis, piriformis, rectus femoris, latissimus dorsi, quadratus lumbo- 
rum y tensor de la fascia lata. Además suelen presentar debilidad de los glúteos, isquiotibiales, y abdominales, los cuales deben fortalecerse debido al rol de estabilidad que dan por originarse el ligamento sacrotuberoso.

La manipulación está indicada en pacientes con sintomatología menor a 17 días, con dolor que no desciende por debajo de la rodilla, hipomovilidad lumbar, y rotación interna de al menos una cadera a $35^{\circ}$. Debe ser realizado por fisioterapeutas de preferencia. ${ }^{12}$ Sin embargo, la manipulación no tiene una metodología estandarizada y ejercicios probados, por lo que los resultados positivos con significancia estadística pueden verse alterados. ${ }^{6}$

\section{Proloterapia}

Terapia que involucra inyección de sustancias no irritativas y no farmacológicas como dextrosa o plasma rico en plaquetas, con el objetivo de iniciar una respuesta inflamatoria que mejore el aporte sanguíneo y la reparación de tejidos acelerada. De igual forma, los estudios suelen no tener la metodología adecuada que se debe tener en cuenta para evitar una malinterpretación de resultados. ${ }^{6}$

En una revisión de Ou-Yang, se han hecho estudios donde comparan las inyecciones de proloterapia con las de corticoesteroides. Aunque refieren que duran más las de proloterapia, son requeridas en mayor cantidad. ${ }^{1}$

Cusi demuestra resultados adecuados con mejoría en escalas funcionales. Los resultados mejoraron desde los tres meses posteriores y la principal mejoría se dio a los 12 meses. El problema es el no haber tenido control con placebo. Debido a ello, se deben tomar los resultados con reservas y esperar a estudios con metodología más estricta. ${ }^{20}$

\section{Rizotomía}

Cuando un paciente con una adecuada respuesta a la inyección intraarticular tiene una respuesta negativa al resto del tratamiento conservador, la rizotomía es el siguiente paso a realizar de tratamiento. También es conocida como la radiofrecuencia de denervación o de ablación. ${ }^{4}$

La rizotomía es un procedimiento donde la corriente entra desde un generador a una punta redondeada. En la punta se forma una esfera de campo electromagnético que lesiona y disemina la energía fuera del cuerpo. Dentro de la rizotomía, existen tres tipos. La radiofrecuencia de baja intensidad se mantiene por 60-90 segundos en un mismo sitio a una tempe- ratura específica; la radiofrecuencia fría incluye una irrigación en la punta de solución salina; y la radiofrecuencia pulsátil, la señal se interrumpe cada medio segundo llegando a una temperatura de $42{ }^{\circ} \mathrm{C} .{ }^{21}$

Otros describen la radiofrecuencia como convencional, donde la energía utilizada puede causar más lesión por temperatura; radiofrecuencia bipolar, donde la energía y la lesión se da únicamente entre las dos puntas del generador, siendo lo óptimo que haya una separación de $6 \mathrm{~mm}$; y la radiofrecuencia fría, que tiene como ventaja poder tener un diámetro de ablación mayor que la convencional, manteniendo los tejidos con temperaturas adecuadas para la ablación sin lesionar excesivamente el tejido circundante. ${ }^{1,6}$

La radiofrecuencia convencional muestra como limitación el fallo de lesión del punto nociceptivo, por ello, se requieren múltiples puntos de ablación. ${ }^{6} \mathrm{Si}$ se compara con las ventajas de la radiofrecuencia bipolar, en donde sí se colocan las ramas laterales del ramo dorsal entre ambas puntas de la radiofrecuencia, entonces se podrá hacer una ablación de todos los puntos nociceptivos. ${ }^{6}$

Las ramas nerviosas que son el objetivo de la rizotomía son la rama medial de L4, ramo dorsal de L5 y ramas laterales del ramo dorsal de S1-S4.

Uno de los principales problemas con la rizotomía de ablación es la variabilidad anatómica por lo que se ha demostrado que el uso de radiofrecuencia bipolar, por su forma de acción, puede detectar hasta el $100 \%$ de todo el ramo lateral sacro. ${ }^{22}$

La radiofrecuencia fría fue comparada con placebo en un estudio por Patel y colaboradores y demuestran resultados favorables a los tres y seis meses en más de la mitad de los pacientes que fueron intervenidos. ${ }^{23}$

Peebles y su equipo determinan mejores resultados con radiofrecuencia fría en pacientes obsesos y con alto nivel de actividad. ${ }^{12}$

Aydin y su grupo concluyen que más de la mitad de los pacientes tendrán una disminución del dolor en más del $50 \%$ en tres meses. A los seis meses el porcentaje de pacientes con disminución del dolor fue de $49 \%$. Sin embargo, se incluyen estudios observacionales retrospectivos y prospectivos. Además las técnicas utilizadas en los distintos análisis fueron variadas. Los propios autores mencionan que para estudios próximos, se requiere incluir más ensayos clínicos aleatorizados. $^{21}$

\section{Cirugía}

Descrita desde 1900, la artrodesis de la articulación sacroiliaca está indicada en pacientes que han fallado 
An Med ABC. 2021; 66 (2): 120-127

al resto de los tratamientos ya mencionados por los últimos seis meses. Se vuelve imperativo descartar otros sitios de lesión o de origen del dolor, lo cual ayuda a identificar las inyecciones intraarticulares. Se puede realizar mediante abordaje anterior, lateral o posterior, con placas o tornillos transiliosacros. . $^{4,12,18}$

Cohen en su revisión logra identificar estudios que reportan un $70 \%$ de éxito en el seguimiento de los casos y otros donde hay un fallo en $82 \%$, por lo que concluye que la indicación quirúrgica no es tan clara como en otras patologías traumáticas. ${ }^{6}$

Rashbaum y colaboradores determinan en su artículo mejoría en las escalas funcionales, escalas de dolor, y de calidad de vida; $87 \%$ con adecuada artrodesis, sin aflojamiento ni migración del material de artrodesis. $^{4}$

Ou-Yang y su equipo reportan la posibilidad de realizar la artrodesis de forma abierta o mínimamente invasiva. Refiere que las complicaciones de la artrodesis abierta se reportan con tromboembolia pulmonar $0-4 \%$, neuropatía postoperatoria $0-2.8 \%$, bursitis transitoria de trocánter mayor $0-2.8 \%$, infección de herida en $4.3 \%$ y necesidad de retiro de material en un $9-25 \%$. $^{1}$

La fusión mínimamente invasiva tiene resultados consistentes con éxito en $85-100 \%$ de los pacientes estudiados. Las complicaciones que pueden presentar son bursitis trocantérica en 1.7-16\%, hematoma $4-16 \%$, infección profunda de $2-11 \%$, y revisión de implantes de 0-6\%. Los resultados adecuados se han reportado hasta por cinco años. La fusión suele darse en un $86-96.9 \%{ }^{1}$

Barros y colaboradores mencionan mejores resultados en casos sometidos a artrodesis mínimamente invasiva a los 12 meses, comparado con la artrodesis abierta. De igual forma, el grupo quirúrgico tuvo mejor control del dolor en un $81.4 \%$, comparado con el $26.1 \%$ de los que fueron tratados de forma conservadora. El bloqueo articular con remisión del dolor en más del $75 \%$ con bloqueo articular es un predictor adecuado de un resultado exitoso en pacientes que serán sometidos a cirugía. Otros predictores son pacientes no fumadores, no consumidores de opioides y ancianos con patología de larga evolución. Las indicaciones para este tratamiento son falla del tratamiento conservador por seis meses, tres pruebas provocativas positivas o más, una remisión de más del $50 \%$ del dolor con inyección intraarticular. ${ }^{17}$

En un estudio aleatorizado se comparó la artrodesis de articulación sacroiliaca con el tratamiento conservador y se llegó a la conslusión de que la cirugía de artrodesis con implantes triangulares transar- ticulares laterales con adecuados resultados en dolor lumbar y miembro pélvico muestra mejoría en calidad de vida, estado de trabajo, distancia de marcha y al realizar pruebas físicas específicas. ${ }^{24}$

\section{CONCLUSIONES}

La patología en la articulación sacroiliaca es todavía una entidad poco conocida por los ortopedistas, sin embargo, el dolor en la región sacrolumbar es uno de los más frecuentes problemas que se ven en la consulta día a día.

En la gran mayoría de las ocasiones está infradiagnosticada. Con esto no se quiere inferir que se hace mal diagnóstico, pero una gran cantidad de casos pueden estar manejados de manera equivocada.

El objetivo de esta revisión es la descripción de la anatomía, así como de la clínica y en especial la exploración física en la que se detallan las pruebas diagnósticas, todo esto con el fin de dar un instrumento más a aquellos médicos interesados para el bien de los pacientes.

\section{REFERENCIAS}

1. Ou-Yang DC, York PJ, Kleck CJ, Patel VV. Diagnosis and management of sacroiliac joint dysfunction. J Bone Joint Surg Am. 2017; 99 (23): 2027-2036. doi: 10.2106/JBJS.17.00245. PMID: 29206793.

2. Thawrani DP, Agabegi SS, Asghar F. Diagnosing sacroiliac joint pain. J Am Acad Orthop Surg. 2019; 27 (3): 85-93. doi: 10.5435/JAAOS-D-17-00132. PMID: 30278010.

3. Palsson TS, Gibson W, Darlow B, Bunzli S, Lehman G, Rabey $\mathrm{M}$ et al. Changing the narrative in diagnosis and management of pain in the sacroiliac joint area. Phys Ther. 2019; 99 (11): 1511-1519. doi: 10.1093/ptj/pzz108. PMID: 31355883.

4. Rashbaum RF, Ohnmeiss DD, Lindley EM, Kitchel SH, Patel VV. Sacroiliac joint pain and its treatment. Clin Spine Surg. 2016; 29 (2): 42-48. doi: 10.1097/BSD.0000000000000359. PMID: 26889985.

5. Vleeming A, Schuenke MD, Masi AT, Carreiro JE, Danneels L, Willard FH. The sacroiliac joint: an overview of its anatomy, function and potential clinical implications. J Anat. 2012; 221 (6): 537-567. doi: 10.1111/j.1469-7580.2012.01564.x. Epub 2012 Sep 19. PMID: 22994881; PMCID: PMC3512279.

6. Cohen SP, Chen Y, Neufeld NJ. Sacroiliac joint pain: a comprehensive review of epidemiology, diagnosis and treatment. Expert Rev Neurother. 2013; 13 (1): 99-116. doi: 10.1586/ern.12.148. PMID: 23253394.

7. Schneider BJ, Rosati R, Zheng P, McCormick ZL. Challenges in diagnosing sacroiliac joint pain: a narrative review. PM R. 2019; 11 Suppl 1: S40-S45. doi: 10.1002/pmrj.12175. Epub 2019 Jul 3. PMID: 31020770.

8. Slobodin G, Rimar D, Boulman N, Kaly L, Rozenbaum M, Rosner I et al. Acute sacroiliitis. Clin Rheumatol. 2016; 35 (4): 851-856. doi: 10.1007/s10067-016-3200-6. Epub 2016 Feb 4. PMID: 26847855.

9. Foley BS, Buschbacher RM. Sacroiliac joint pain: anatomy, biomechanics, diagnosis, and treatment. Am J Phys 
Med Rehabil. 2006; 85 (12): 997-1006. doi: 10.1097/01. phm.0000247633.68694.c1. PMID: 17117004.

10. Nejati P, Sartaj E, Imani F, Moeineddin R, Nejati L, Safavi M. Accuracy of the diagnostic tests of sacroiliac joint dysfunction. J Chiropr Med. 2020; 19 (1): 28-37. doi: 10.1016/j.jcm.2019.12.002. Epub 2020 Sep 12. PMID: 33192189; PMCID: PMC7646135.

11. Laslett M, Aprill CN, McDonald B, Young SB. Diagnosis of sacroiliac joint pain: validity of individual provocation tests and composites of tests. Man Ther. 2005; 10 (3): 207-218. doi: 10.1016/j.math.2005.01.003. PMID: 16038856 .

12. Peebles R, Jonas CE. Sacroiliac joint dysfunction in the athlete: diagnosis and management. Curr Sports Med Rep. 2017; 16 (5): 336-342. doi: 10.1249/JSR.0000000000000410. PMID: 28902756.

13. Soto Quijano DA, Otero Loperena E. Sacroiliac joint interventions. Phys Med Rehabil Clin N Am. 2018; 29 (1): 171-183. doi: 10.1016/j.pmr.2017.09.004. PMID: 29173661.

14. Schneider BJ, Ehsanian R, Rosati R, Huynh L, Levin J, Kennedy DJ. Validity of physical exam maneuvers in the diagnosis of sacroiliac joint pathology. Pain Med. 2020; 21 (2): 255-260. doi: 10.1093/pm/pnz183. Erratum in: Pain Med. 2020; 21 (5): 1089. PMID: 31393577.

15. Simopoulos TT, Manchikanti L, Gupta S, Aydin SM, Kim CH, Solanki D et al. Systematic review of the diagnostic accuracy and therapeutic effectiveness of sacroiliac joint interventions. Pain Physician. 2015; 18 (5): E713-E756. PMID: 26431129.

16. Taheri A, Lajevardi M, Abad M, Zebardast J, Shaabani S. Sacroiliac joint intraarticular injection in true anteroposterior view: description of a new C-Arm guided method. Pain Physician. 2018; 21 (1): 61-66. PMID: 29357334.

17. Barros G, McGrath L, Gelfenbeyn M. Sacroiliac joint dysfunction in patients with low back pain. Fed Pract. 2019; 36 (8): 370-375. PMID: 31456628; PMCID: PMC6707638.
18. Dreyfuss P, Dreyer SJ, Cole A, Mayo K. Sacroiliac joint pain. J Am Acad Orthop Surg. 2004; 12 (4): 255-265. doi: 10.5435/00124635-200407000-00006. PMID: 15473677.

19. Kamali F, Zamanlou M, Ghanbari A, Alipour A, Bervis S. Comparison of manipulation and stabilization exercises in patients with sacroiliac joint dysfunction patients: A randomized clinical trial. J Bodyw Mov Ther. 2019; 23 (1): 177-182. doi: 10.1016/j.jbmt.2018.01.014. Epub 2018 Jan 31. PMID: 30691749.

20. Cusi M, Saunders J, Hungerford B, Wisbey-Roth T, Lucas P, Wilson $\mathrm{S}$. The use of prolotherapy in the sacroiliac joint. Br J Sports Med. 2010; 44 (2): 100-104. doi: 10.1136/ bjsm.2007.042044. Epub 2008 Apr 9. PMID: 18400878.

21. Aydin SM, Gharibo CG, Mehnert M, Stitik TP. The role of radiofrequency ablation for sacroiliac joint pain: a meta-analysis. PM R. 2010; 2 (9): 842-851. doi: 10.1016/j. pmrj.2010.03.035. PMID: 20869684.

22. Yang AJ, McCormick ZL, Zheng PZ, Schneider BJ. Radiofrequency ablation for posterior sacroiliac joint complex pain: a narrative review. PM R. 2019; 11 Suppl 1: S105-S113. doi: 10.1002/pmrj.12200. Epub 2019 Jul 25. PMID: 31169356.

23. Patel N, Gross A, Brown L, Gekht G. A randomized, placebocontrolled study to assess the efficacy of lateral branch neurotomy for chronic sacroiliac joint pain. Pain Med. 2012; 13 (3): 383-398. doi: 10.1111/j.1526-4637.2012.01328.x. Epub 2012 Feb 2. PMID: 22299761.

24. Dengler J, Kools D, Pflugmacher R, Gasbarrini A, Prestamburgo D, Gaetani P et al. Randomized trial of sacroiliac joint arthrodesis compared with conservative management for chronic low back pain attributed to the sacroiliac joint. J Bone Joint Surg Am. 2019; 101 (5): 400411. doi: 10.2106/JBJS.18.00022. PMID: 30845034; PMCID: PMC6467578. 